\title{
The Relationship between Perceived Health Message Motivation and Social Cognitive Beliefs in Persuasive Health Communication
}

\author{
Kiemute Oyibo ${ }^{1,2}$ (D) \\ 1 School of Public Health and Health Systems, University of Waterloo, Waterloo, ON N2L 3G1, Canada; \\ kiemute.oyibo@usask.ca \\ 2 Department of Computer Science, University of Saskatchewan, Saskatoon, SK S7N 5C9, Canada
}

Citation: Oyibo, K. The Relationship between Perceived Health Message Motivation and Social Cognitive Beliefs in Persuasive Health Communication. Information 2021, 12, 350. https://doi.org/10.3390/ info12090350

\section{Academic Editor:}

Ognjen Arandjelović

Received: 1 July 2021

Accepted: 21 August 2021

Published: 28 August 2021

Publisher's Note: MDPI stays neutral with regard to jurisdictional claims in published maps and institutional affiliations.

Copyright: (C) 2021 by the author. Licensee MDPI, Basel, Switzerland. This article is an open access article distributed under the terms and conditions of the Creative Commons Attribution (CC BY) license (https:// creativecommons.org/licenses/by/ $4.0 /)$.

\begin{abstract}
People respond to different types of health messages in persuasive health communication aimed at motivating behavior change. Hence, in human factors design, there is a need to tailor health applications to different user groups rather than change the human characteristics and conditions. However, in the domain of fitness app design, there is limited research on the relationship between users' perceived motivation of health messages and their social-cognitive beliefs about exercise, and how this relationship is moderated by gender. Knowledge of the gender difference will help in tailoring fitness apps to the two main gender types. Hence, I conducted an empirical study to investigate the types of health messages that are most likely to motivate users and how these messages are related to outcome expectation, self-efficacy, and self-regulation beliefs in the context of exercise modeling. The results of the data analysis show that users are more motivated by illness- and death-related messages compared with obesity-, social stigma-, and financial cost-related messages. Moreover, illnessand death-related messages have a significant relationship with users' social-cognitive beliefs about bodyweight exercise. These findings indicate that, in the fitness domain, illness- and death-related messages may be employed as a persuasive technique to motivate regular exercise.
\end{abstract}

Keywords: health message; persuasive communication; persuasive technology; social cognitive belief; fitness app

\section{Introduction}

With the advent of the smartphone, public health communication has become popular. It seeks to promote and protect the health of the general population through persuasive health messages. The messages aim to influence social norms, advocate health-centered public policy, increase individual knowledge and awareness, and promote behaviors beneficial to the target audience [1]. In public health communication campaigns and interventions, persuasive messages are sent to the public to elicit certain health outcomes, including performance of healthy behaviors such as healthy eating, physical activity, and smoking cessation. For such messages to be effective, certain guidelines are often followed by experts, including determining the target audience, the expected responses, the specific message to be sent, evidence to support the message, and the type of appeal (negative or positive) to be used. Effective health messages are designed to reflect the interests, preferences, and values of the target audience. They have the potential to quickly reach a large audience and elicit the target behaviors [1].

However, there is limited research on the relationship between health messages and the target users' social-cognitive beliefs, which are determinants of health behaviors. Moreover, there are no studies on the moderating effect of key demographic characteristics, even though public health communication campaigns that "tailor health messages to recipients' sociodemographic characteristics are more effective than uniform ones" (p. 11) [2]. To bridge this gap, I conducted a large-scale empirical study $(n=669)$ to 
investigate the relationships between users' perceived motivation of health messages and their social-cognitive-beliefs when observing behavior models performing bodyweight exercise; and how gender moderates the relationships. On one hand, I focused on five types of health messages: obesity-, social stigma-, financial cost-, illness-, and death-related. On the other hand, I focused on three social-cognitive beliefs: self-efficacy, self-regulation, and outcome expectation.

The data analysis shows that, regardless of gender, users are more likely to be motivated by illness- and death-related messages compared with financial-cost-, obesity- and social-stigma-related messages. I found a significant relationship between users' perceived motivation of illness- and death-related messages and their self-regulation beliefs. I also found a significant relationship between users' perceived motivation of illness-related messages and their outcome expectations and self-efficacy beliefs. Particularly, I found a significant relationship between females' perceived motivation of social-stigma-related messages and their outcome expectations and self-efficacy beliefs, but none for males. The findings indicate that illness- and death-related messages are most likely to be effective in motivating behaviors in the fitness domain.

\section{Background}

In this paper, I aim to achieve two objectives. The first objective is to investigate the relationship between users' perceived motivation of health messages and social-cognitive beliefs about bodyweight exercise, and the moderating effect of gender. The second objective is to examine users' levels of perceived motivation with regard to different types of health messages and the effect of gender. Hence, I provide an overview of persuasive messages, behavior modeling, and social cognitive theory (SCT) in the context of persuasive health communication.

\subsection{Health Communication}

Health communication links the fields of communication and health [3]. It is the study and use of communication strategies to inform a group of individuals and enhance their health [4]. Typical channels for health communication include flyers, brochures, newspaper, radio, television, emails, mobile apps, social media, and websites [5,6]. Health communication has "relevance for virtually every aspect of health and well-being, including disease prevention, health promotion and quality of life" [7]. Hence, it is "increasingly [being] recognized as a necessary element of efforts to improve personal and public health" [3]. Particularly, health communication is used as a persuasive technique for motivating behavior change. This entails wording, phrasing, framing and tailoring health messages in ways that are more effective in eliciting the target behaviors.

Four key health communication techniques that have been identified to support and motivate behavior change include personalization, tailoring, narrative, and framing. Personalization is health communication designed for an individual based on information gathered from the individual, especially while communication with a health application. Tailoring (also known as targeting) is health communication designed for groups of people based on demographic characteristics such as age, gender, sex, race, culture, language, or psychographic characteristics such as attitude, motivation, interest, lifestyle, or personality traits. Narrative is health communication delivered to the target audience in the form of a story, testimonial, or entertainment-based education [3]. Finally, framing is the communication of the same health message in alternate ways. Examples of message framing include gain vs. loss, rational vs. emotional, etc. Specifically, rational messages appeal to logic, while emotional messages appeal to emotion (e.g., by highlighting fear and severity). Rational appeal works better with an audience that is interested in the target behavior, while emotional appeal works better with an audience that is not already interested in the target behavior [8]. Moreover, gain-based framing is the phrasing of a statement in terms of its positive (gain) features and/or outcomes, while loss-based framing is the phrasing of a statement in terms of its negative (loss) features and/or outcomes [9]. 
One of the main fields of health communication where communication techniques are utilized to motivate behavior change is mobile health (mHealth, for short). MHealth is the "use of mobile and wireless technologies to support the achievement of health objectives" (p. 1) [10]. As stated by the World Health Organization [10], mHealth has the potential to revolutionize healthcare service delivery across the globe. Particularly, mobile phones (including feature and smart phones) have become a very popular and powerful health communication medium and channel for healthcare service delivery. Through the power of the mobile phone, more and more people are becoming only one click away from accessing health information and/or services. By means of short message service (SMS) and mobile health apps, tailored information and intervention materials are disseminated to specific target audiences with the intent of spreading knowledge, supporting, and motivating behavior change [3]. A systematic review by Hall et al. [11] found that most text-messaging interventions were effective in addressing health problems in domains such as physical activity, smoking cessation, weight management, diabetes self-management, and medication adherence for antiretroviral therapy. Particularly, in recent years, fitness apps have gained traction as a persuasive technology for motivating physical activity. Fitness apps are being used by different individuals to meet different health goals such as setting exercise goals, developing exercise routines, increasing motivation/self-awareness, and/or improving eating habits [12,13]. This has sparked intensive research into the potential effectiveness of fitness apps in fostering behavior change [14-17]. However, there is limited work on the relationship between the perceived motivation of health messages and social-cognitive beliefs in the context of exercise modeling. Investigating this relationship will help designers of physical activity interventions know the types of messages that should be targeted at improving social-cognitive determinants of exercise. This social-cognitive determinants (e.g., self-efficacy, self-regulation, and outcome expectation) have the potential of motivating behavior change in the physical domain $[18,19]$.

\subsection{Behavior Modeling}

Behavior modeling is a persuasive technique, in which an expert, coach or teacher demonstrates to an observer the correct performance of a given behavior [20]. In the context of social learning, the demonstration of the behavior helps the observer to perform it easily and correctly. A typical example of health behaviors modeled by human-computer-interaction technologies (such as virtual coaches [21] and humanoid robots [22]) is bodyweight exercise aimed at improving the physical and mental health of the observer of the behavior. In a systematic review by Conroy et al. [23], behavior modeling (based on instructions and demonstrations) was found to be one of the most commonly employed persuasive strategies in fitness apps on the market.

\subsection{Social Cognitive Theory}

The SCT is a behavior change theory that helps in explaining why people engage in certain behaviors. It posits that human cognition, the environment, and behavior interact reciprocally to define and/or shape behaviors [24]. The key cognitive factors found to determine behaviors include self-efficacy, self-regulation, and outcome expectation [25,26]. These cognitive factors interact with environmental factors such as social and technological systems to shape human behaviors. For example, a mobile app featuring exercise models (e.g., a personal trainer or virtual coach) is a socio-technical system aimed at motivating behavior change using mobile technology as an information communication medium. As stated by Bandura [24], socio-technical systems, such as persuasive technologies, can influence human behaviors, with social-cognitive beliefs such as self-efficacy, self-regulation, and outcome expectation acting as mediators and/or proximal determinants of the target behavior [27-29]. The effectiveness of personalizing health messages to individuals "will depend on whether weak or strong determinants are targeted" (p. 11) [2]. In this study, I focused on the three common social-cognitive factors (self-efficacy, self-regulation, and 
outcome expectation), which, research has shown, are strong mediators and/or direct determinants of behavior change [19,30,31].

Self-efficacy is the belief in one's ability to perform a given behavior. Moreover, it is the feeling of a sense of control over one's behavior and the environment in which it is carried out [32,33]. In the SCT model, it is found to be the strongest (proximal) determinant of behavior [25].

Self-regulation is the management of one's thoughts, feelings and actions towards the achievement of a goal. It entails setting specific and measurable goals, making plans, and monitoring their achievement. In the SCT model, self-regulation directly influences behavior, with cognitive beliefs such as self-efficacy and outcome expectation acting as mediators [31,34].

Finally, outcome expectation is the belief one holds about the outcomes of behavior. It entails the costs and benefits derived from the (non-)performance of a behavior, which could be social, physical, or mental, in the context of physical activity. In the SCT model, outcome expectation has a direct influence on behavior and/or mediate the influence of social-cognitive beliefs such as self-efficacy on the target behavior [25,35].

\subsection{Gender-Based Tailoring}

Research shows that gender can be leveraged as a mechanism for tailoring humancomputer interaction systems to make them more effective [36,37]. In persuasive technology, there is a plethora of evidence that males and females differ significantly in terms of the persuasive strategies to which they are more likely to respond. For example, in the healthy eating domain, Orji et al. [38] found that females are more persuadable by persuasive strategies such as cooperation, customization, and praise than males. Moreover, Oyibo et al. [39] found that males are more persuadable by competition and reward than females, especially in collectivist cultures. Similarly, in the education domain, Abdullahi et al. [40] found that males are more persuadable by social learning and reward than females, while females are more persuadable by trustworthiness than males. Finally, in path modeling research, Oyibo and Vassileva [41] found that the relationship between reward and competition is stronger for males than females, while the relationship between reward and social learning is stronger for females than males. These prior findings in persuasive technology research are an indication that males and females may differ with regard to the perceived motivation of health-related messages and in the relationship between perceived motivation of health messages and social-cognitive beliefs.

\section{Method}

In this section, I focus on the research objectives, research design, measurement instruments, and study participants.

\subsection{Research Objectives}

In this study, I aim to achieve two main objectives, each of which comprises two components. The first objective is to investigate: (a) the relationship between users' perceived motivation of different types of health messages and their social-cognitive beliefs about exercise; and (b) the moderating effect of gender. The second objective is to examine: (a) users' perceived motivation of different types of health messages; and (b) the effect of gender on the perceived motivation profile. Perceived health message motivation, in the context of this study, is the degree to which a user is motivated by a given health message to begin or continue exercising. This is similar to perceived motivation in the education domain. For example, Information Use, a subscale of Academic Motivation Scale, is defined as "the motivation to learn new and different things, and to use them both inside and outside school" (p. 25) [42].

The study is important because little attention has been paid to the relationship between users' perceived motivation of different types of health messages and their social-cognitive beliefs in the context of exercise modeling, which is one of the most employed behavior 
change techniques in fitness apps on the market [23]. In particular, there is little to no research in the fitness app domain on whether negatively framed health messages will positively or negatively impact the social-cognitive beliefs of observers of exercise models. Most of the research regarding threat-based messages have been in the domain of smoking cessation, texting-while-driving, and risky sexual behavior [43-45]. In these domains, threat-based messages (especially those related to death) are discouraged given that they may elicit active coping defenses in the target subjects. For example, as stated by Martin and Kamins [43], "when smokers are faced with thoughts of death through shocking images, they produce active coping defenses as reflected in a boomerang effect" (p. 10). Hence, there is a need for similar research, especially exploratory, in the fitness domain given that fitness apps have become very popular due to the advancement of mobile technology in the last decade, which has resulted in the creation and ubiquity of the smartphone.

To achieve the research objectives, I prototyped a fitness app in which a fitness expert demonstrates to the user how to correctly perform a bodyweight exercise (Figure 1). I considered gender (male and female), race (black and white) and exercise type (push-up and squat) [46]. The current study does not investigate the effect of these variables.
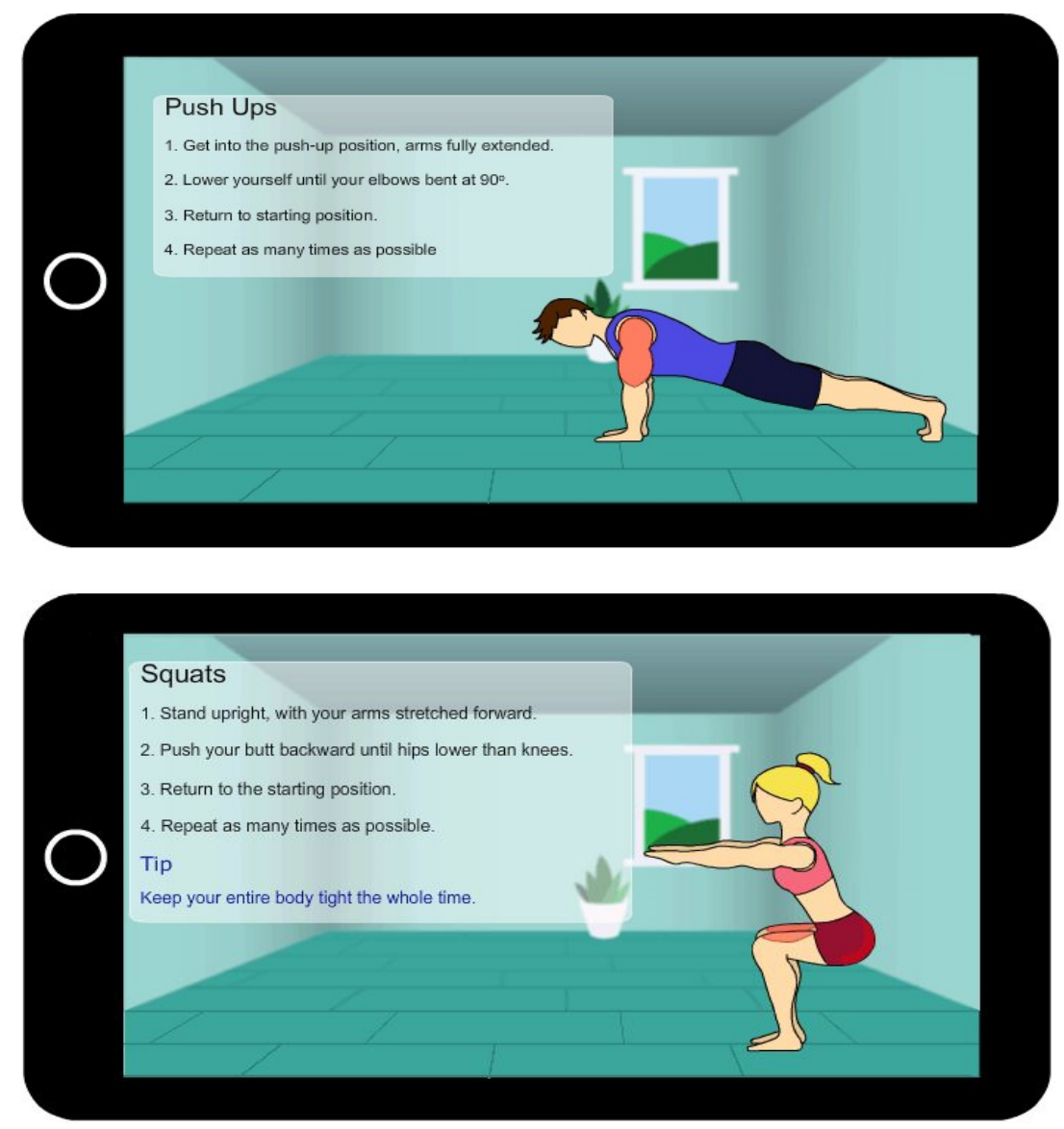

Figure 1. Two of the eight behavior models for push-up and squat bodyweight exercise [20].

The study is based on a quantitative approach. In an online survey, I asked participants questions on five different types of health messages related to obesity, social stigma, money, illness, and death. The questions are drawn from the literature as shown in Table 1. The framing of the messages focuses on negative outcomes of physical inactivity. Research shows that negatively framed messages are more likely to be effective than positively framed messages, especially when people evaluate them carefully [47]. I chose these five types of health messages, which focus on obesity, financial loss, illness, death, and social stigma, because they are among the common consequences associated with physical 
inactivity. Secondly, the messages attempted to satisfy McGuire's [8] key criteria for designing effective messages, which are:

1. Ensure the message is clear.

2. Ensure the message is credible, believable and realistic.

3. Provide good evidence for costs (threats) and benefits for not doing and doing the target behavior, respectively.

4. Ensure the message uses an appeal that is appropriate for the target audience.

5. Ensure the messenger is seen as a credible source of the information.

6. Ensure the message will not be offensive or harm the target audience.

7. Ensure the behavior the target audience is asked to perform is reasonably easy.

8. Ensure the message gets and maintains the attention of the target audience.

Table 1. Measurement instruments.

Please kindly rate the following messages below:

(1) Financial cost-related [48]: "Physical inactivity costs Canadian tax payers $\$ 6.8$ billion a year' (Source: CBCNews)."

(2) Obesity-related [49]: "One in four Canadian adults has clinical obesity (Source: Canadian Institute for Health Information)."

Health Message [Does not motivate me to start or continue exercising (1) to Completely motivates me to start or continue exercising (7)]

\section{Self-Efficacy}

[Not Confident (0) to Confident (100)] [33]

Outcome

Expectation

[Strongly Disagree (1) to

Strongly Agree (5)] [35]

Self-Regulation [Strongly Disagree (1) to Strongly Agree (5)] [31]
(3) Death-related [50]: " $6 \%$ of the world's death is caused by physical inactivity' (Source: World Health Organization)."

(4) Illness-related [51]: "Those who do not find time for exercise will have to find time for illness' (Source: Edward Stanley)."

(5) Social stigma-related [52]: "The stigma against people with obesity is comparable to that of racial discrimination' (Source: Canadian Obesity Network)."

How confident are you that you can complete at home the proposed weekly number of push-ups (entered previously) for the next 3 months.

(1) Even when you have worries and problems?

(2) Even if you feel depressed?

(3) Even when you feel tense?

(4) Even when you are tired?

(5) Even when you are busy?

The [name of exercise] will ...

(1) Improve my ability to perform daily activities.

(2) Improve my overall body functioning.

(3) Strengthen my bones.

(4) Increase my muscle strength.

(5) Improve the functioning of my cardiovascular system.

(6) Improve my social standing.

(7) Make me more at ease with people.

(8) Increase my acceptance by others.

To achieve my proposed weekly average number of push-ups ...

(1) I will set a goal.

(2) I will develop a series of steps to reach my weekly goal.

(3) I will keep track of my progress in meeting my goal.

(4) I will endeavor to achieve the set goal for myself.

(5) I will make my goal public by telling others about it.

Particularly, regarding the third and fourth criteria, since the fitness app is aimed at promoting exercise, I focused on threat-based rational/emotional messages that emphasize the cost of physical inactivity, which could be financial, social, health-related, etc. Regarding the fifth criterion, I focused on factual and credible messages by citing their source. For example, the death-related message, " $6 \%$ of the world's death is caused by physical inactivity," is a threat-based message that is likely to be perceived as a credible message 
given that the cited source (World Health Organization) is an authority on health issues. Regarding the seventh criterion, the target behavior (start or continue exercising) being reasonably easy is captured in the response part of the question related to the health message: "does not motivate me to start or continue exercising (1) or completely motivates me to start or continue exercising (7)." Regarding the eighth criterion, I investigated whether the five messages are able to motivate users to engage in exercise [8].

In addition, I asked participants questions about their social-cognitive beliefs about the modeled bodyweight exercise. The questions are related to self-efficacy, self-regulation, and outcome expectation as shown in Table 1.

\subsection{Research Questions}

The research questions I aim to answer in this study on health messages, exercise modeling, and social-cognitive beliefs are as follows:

RQ1. Is there a relationship between users' perceived motivation of health messages and social-cognitive determinants of exercise?

RQ2. What is the users' perceived motivation profile regarding financial-cost-, illness-, obesity-, stigma- and death-related health messages?

RQ3. Are the relationships between users' perceived health message motivation and social-cognitive beliefs moderated by gender?

RQ4. Does users' perceived motivation of health messages vary across gender?

\subsection{Exploratory Model}

Figure 2 shows the exploratory model on the relationship between perceived motivation of health messages and social-cognitive beliefs. The model is based on the rational model of persuasion, which states that "people think and behave in ways based on reasons and are relatively predictable." The model postulates that, in the persuasive process, people make use of following rational model [53]:

Belief + Values $/$ Motives $=$ Attitudes $\rightarrow$ Behavior

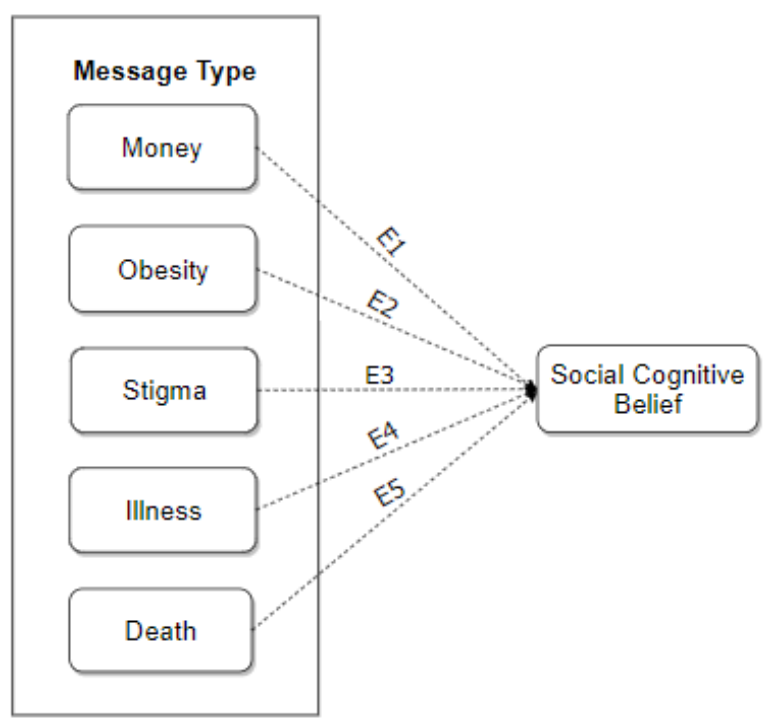

Figure 2. Exploratory research model.

The model holds that beliefs, combined with values (and/or motives), produce attitudes, which in turn influence behavior. Based on this model, I hypothesize that if users believe that a health message is true (i.e., it is a fact), and are motivated by it, both their belief and motivation will influence their social-cognitive beliefs such as perceived self-efficacy, perceived self-regulation, and outcome expectation (regarding bodyweight exercise), which are determinants of physical activity [30]. 


\subsection{Participants}

The study was approved by the Behavior Research Ethics Board of University of Saskatchewan. I recruited participants on Amazon Mechanical Turk [54]. Each participant was remunerated with US $\$ 0.6$. Table 2 shows their demographic information and the randomized distribution of the eight versions of exercise models among the participants. A total of 669 participants took part in the study: 517 were white and 152 were black/brown.

Table 2. Participants' demographics. $\mathrm{BB}=$ black/brown observers of Black models, $\mathrm{WW}=$ white observers of white models, $\mathrm{BW}=$ black/brown observers of white models; $\mathrm{WB}=$ white observers of black models.

\begin{tabular}{|c|c|c|c|}
\hline Criterion & Subgroup & Number & Percent \\
\hline \multirow{2}{*}{ Gender } & Female & 327 & $48.9 \%$ \\
\hline & Male & 342 & $51.1 \%$ \\
\hline \multirow{5}{*}{ Age } & $18-24$ & 126 & $18.8 \%$ \\
\hline & $25-34$ & 296 & $44.2 \%$ \\
\hline & $35-44$ & 155 & $23.2 \%$ \\
\hline & $45-54$ & 60 & $9.0 \%$ \\
\hline & $54+$ & 32 & $4.8 \%$ \\
\hline \multirow{7}{*}{ Education } & Tech/Trade School & 86 & $12.9 \%$ \\
\hline & High School & 136 & $20.3 \%$ \\
\hline & $\mathrm{BSc}$ & 316 & $47.2 \%$ \\
\hline & MSc & 96 & $14.3 \%$ \\
\hline & $\mathrm{PhD}$ & 15 & $2.2 \%$ \\
\hline & Others & 20 & $3.0 \%$ \\
\hline & Canada & 214 & $32.0 \%$ \\
\hline \multirow{2}{*}{$\begin{array}{l}\text { Country of } \\
\text { Origin }\end{array}$} & United States & 378 & $56.5 \%$ \\
\hline & Others & 77 & $11.5 \%$ \\
\hline \multirow{3}{*}{ Race } & Black & 52 & $7.8 \%$ \\
\hline & Brown & 99 & $14.8 \%$ \\
\hline & White & 518 & $77.4 \%$ \\
\hline \multirow{4}{*}{$\begin{array}{l}\text { Observer- } \\
\text { Model Race }\end{array}$} & $\mathrm{BB}$ & 74 & $11.1 \%$ \\
\hline & BW & 78 & $11.7 \%$ \\
\hline & WW & 255 & $38.1 \%$ \\
\hline & WB & 262 & $39.2 \%$ \\
\hline \multirow{4}{*}{ Years on the Internet } & $0-3$ & 4 & $0.6 \%$ \\
\hline & $4-6$ & 31 & $4.6 \%$ \\
\hline & $7-9$ & 60 & $9.0 \%$ \\
\hline & $>10$ & 574 & $85.8 \%$ \\
\hline
\end{tabular}

\section{Results}

This section covers the path modeling of the relationship between perceived health message motivation and social-cognitive beliefs, multigroup analysis, reliability analysis of the measures, and the analysis of variance based on gender and message type.

\subsection{Path Modeling}

Before analyzing the structural models, I evaluated the measurement models to ensure the preconditions for path modeling were met. For the multiple-item constructs, regarding indicator reliability, over $80 \%$ of the indicators had an outer loading on their respective constructs that was greater than 0.7 , while less than $20 \%$ range from 0.45 to 0.70 . Regarding internal consistency reliability, the Dillon-Goldstein metric (DG.rho) for each construct was greater than 0.7 . Regarding convergent validity, the average variance extracted (AVE) for each construct was greater than 0.5. Finally, regarding discriminant validity, the crossloading criterion was used, and no indicator loaded higher on any other construct than the one it measured $[55,56]$. 
Figure 3 shows the path models of the relationship between the message types and each of the social-cognitive-belief constructs. Overall, the models have a goodness of fit (GOF) ranging from 28 to $32 \%$. For the self-efficacy, self-regulation and outcome expectation models, the GOFs are $29 \%, 32 \%$, and $28 \%$, respectively. These values indicate that the overall validation of each of the models is medium [57]. Secondly, the message types account for $11 \%, 17 \%$, and $18 \%$ of the variance of perceived self-efficacy, self-regulation, and outcome expectation, respectively. These coefficient of determination $\left(R^{2}\right)$ values are regarded as low in path modeling [55], suggesting that there are variables, other than users' perceived health message motivation, that account for the variance of the social-cognitive-belief constructs. In the three models, illness- and death-related messages have a significant relationship with each of the three social-cognitive beliefs $(\beta>0.10, p<0.05)$, except that the relationship between the death-related message and self-efficacy belief $(\beta=0.12, p>0.05)$ is non-significant. Overall, the illness-related message, followed by the death-related message, is more consistent in influencing and accounting for the variance of the target construct. It turns out that, in the overall models, financial cost-, obesity-, and stigma-related messages have no significant relationship with the three social-cognitive-belief constructs.

Table 3 shows the results of the multigroup analysis, in which some of the relationships are moderated by gender. Regarding self-efficacy, the social stigma-based relationship is significant in the female model $(\beta=0.17, p<0.05)$, but non-significant in the male model ( $\beta=0.02, p>0.05)$, with the numerical difference being statistically significant $(p<0.05)$. Similarly, regarding outcome expectation, the social stigma-based relationship is significant in the female model $(\beta=0.14, p<0.01)$, but non-significant in the male model $(\beta=0.08$, $p>0.05)$, with the numerical difference being statistically significant $(p<0.05)$.

Table 3. Multigroup analysis for each social-cognitive-belief construct based on gender. $M=M a l e, F=F e m a l e$. Sig = Significance, $\mathrm{n} . \mathrm{s}=$ non-significant, ${ }^{*} p<0.05,{ }^{* * *} p<0.001$.

\begin{tabular}{|c|c|c|c|c|c|c|c|c|c|}
\hline \multirow{2}{*}{$\begin{array}{l}\text { Message } \\
\text { Type }\end{array}$} & \multicolumn{3}{|c|}{ Self-Efficacy } & \multicolumn{3}{|c|}{ Self-Regulation } & \multicolumn{3}{|c|}{ Outcome Expectation } \\
\hline & $\mathbf{M}$ & $\mathbf{F}$ & Sig & $\mathbf{M}$ & $\mathbf{F}$ & Sig & $\mathbf{M}$ & $\mathbf{F}$ & Sig \\
\hline Money & 0.14 & 0.15 & $p<0.05$ & 0.08 & 0.00 & n.s & 0.10 & 0.06 & n.s \\
\hline Obesity & 0.07 & -0.04 & n.s & 0.06 & 0.05 & n.s & 0.06 & 0.21 & n.s \\
\hline Illness & $0.16^{*}$ & 0.11 & n.s & $0.20 *$ & $0.16^{*}$ & n.s & $0.17^{*}$ & $0.03 *$ & n.s \\
\hline Death & 0.12 & 0.06 & n.s & $0.24 *$ & $0.21 *$ & n.s & 0.19 * & 0.13 & 0.06 \\
\hline Stigma & 0.02 & $0.17^{*}$ & $p<0.05$ & 0.04 & 0.11 & n.s & 0.08 & $0.14^{* * *}$ & $p<0.05$ \\
\hline $\mathrm{R}^{2}$ & $14 \%$ & $13 \%$ & & $22 \%$ & $18 \%$ & & $21 \%$ & $18 \%$ & \\
\hline GOF & $33 \%$ & $31 \%$ & & $32 \%$ & $36 \%$ & & $31 \%$ & $28 \%$ & \\
\hline
\end{tabular}




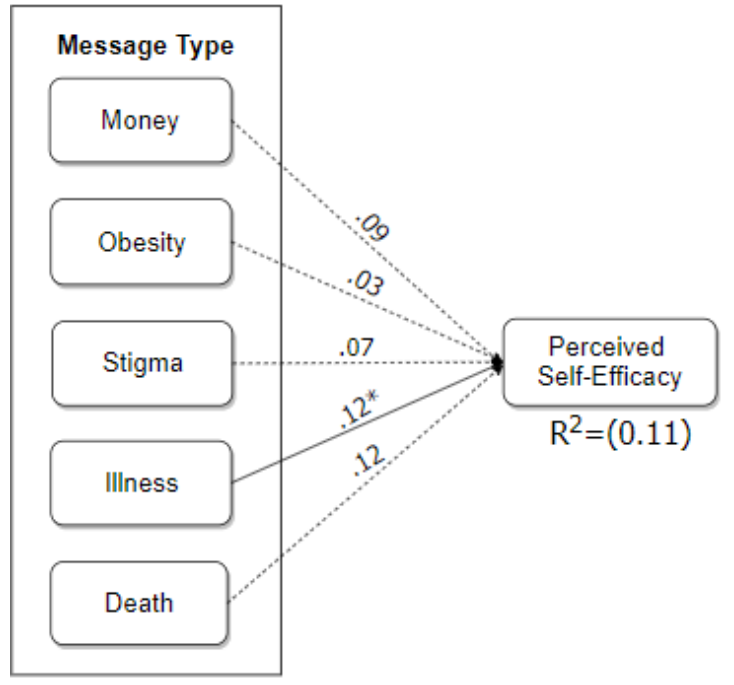

(a)

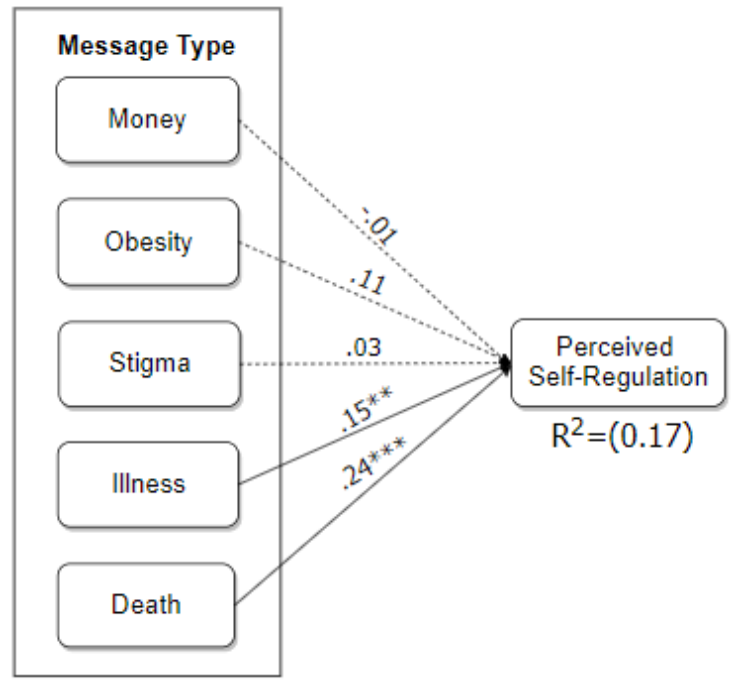

(b)

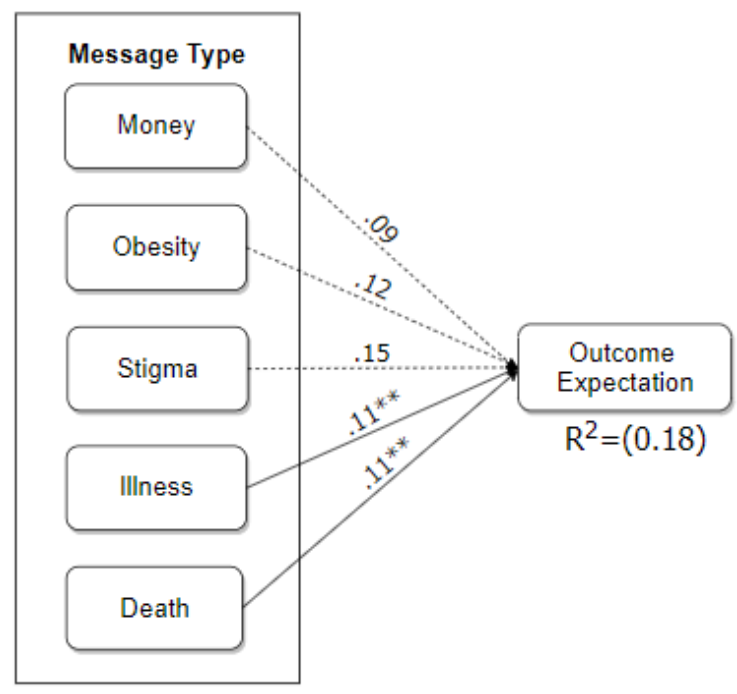

(c)

Figure 3. Model of the relationship between perceived health message motivation and social-cognitive beliefs: (a) self-efficacy, (b) self-regulation, and (c) outcome expectation, ${ }^{*} p<0.05,{ }^{* *} p<0.01,{ }^{* * *} p<0.001$.

\subsection{Analysis of Variance}

Figure 4 shows the perceived motivation profile for the five message types. Due to the non-normality of the dataset, to ensure that the social-cognitive-belief constructs were reliably measured, I performed a non-parametric McDonald's omega reliability test $[58,59]$. The results for each of the social-cognitive-belief constructs satisfied the reliability requirement: omega $(\omega)$ being greater than 0.70 . Thereafter, I carried out a two-way analysis of variance to uncover the effect of gender and message type on users' perceived motivation of health messages. The result of the two-way analysis of variance showed that there is a main effect of gender $[\mathrm{F}(1,3335)=12.30, p<0.001]$ and message type $[\mathrm{F}(4,3335)=12.30, p<0.001]$, but no interaction $[\mathrm{F}(4,3335)=1.16, p>0.05]$ between both factors. Overall, males $(\mathrm{M}=4.19)$ are significantly more receptive $(p<0.01)$ to the health messages than females $(M=3.93)$. Moreover, users are significantly more motivated by the illness-related message $(\mathrm{M}=4.84, \mathrm{SD}=1.80)$ and death-related message $(\mathrm{M}=4.69$, 
$\mathrm{SD}=1.91)$ than the financial-cost-related message $(\mathrm{M}=3.70, \mathrm{SD}=1.94)$, obesity-related message $(\mathrm{M}=3.50, \mathrm{SD}=1.99)$, and stigma-related message $(\mathrm{M}=3.48, \mathrm{SD}=1.95)$.

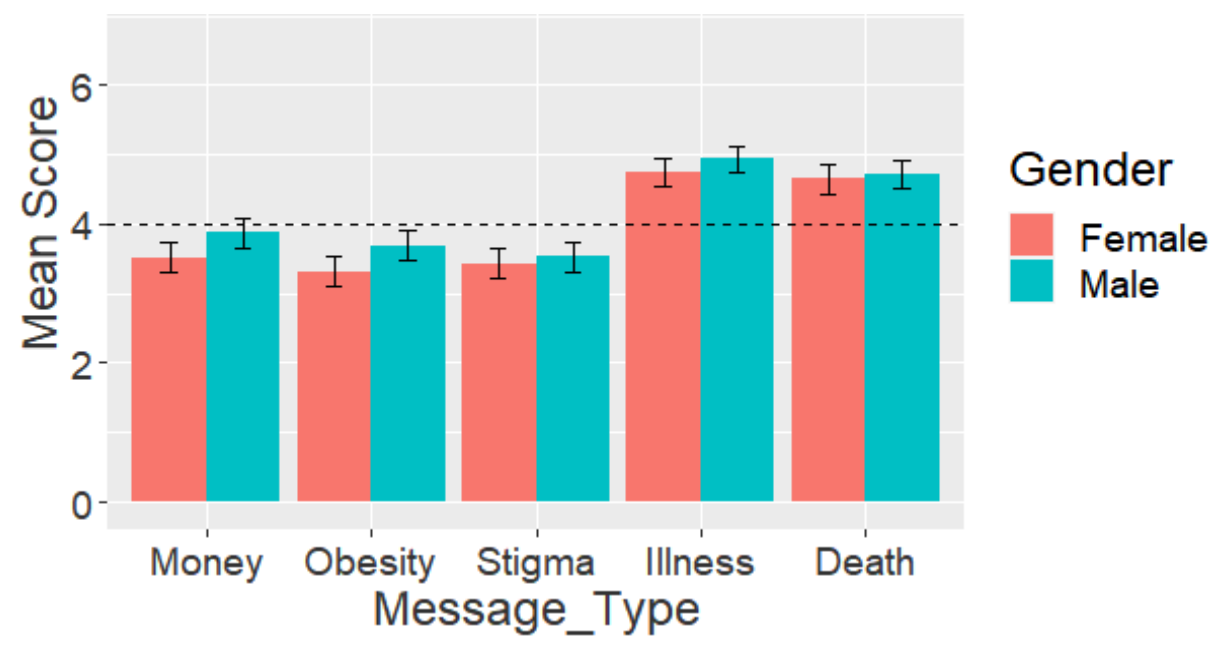

Figure 4. Mean levels of perceived health message motivation on a seven-point scale. The crossbar represents neutral value and the vertical bar represents $95 \%$ confidence interval.

\section{Discussion}

Persuasive messages in health interventions are more likely to be effective if they are tailored to the psychosocial attributes of users [2]. Hence, the need to investigate what types of persuasive messages are likely to be effective and their relationships with the psychological determinants of behaviors. The results of the data analysis show that there is a significant relationship between users' perceived motivation of illness- and death-related messages and their social-cognitive beliefs about exercise. Overall, the path model of the relationship between both constructs (Figure 3 ) has a medium goodness of fit ranging from 28 to $36 \%$, indicating that the overall validation of the model by the data is moderate [57]. Secondly, users' perceived motivation of the health messages accounts for between 11 and $22 \%$ of the variance of the social-cognitive-belief constructs, with illness-, followed by death-, and stigma-related messages, being the most consistent determinants (Table 3). The $\mathrm{R}^{2}$ values is less than $30 \%$ are regarded as low in path modeling [55]. These low values indicate that there are other important variables (e.g., perceived persuasiveness of exercise model [46]) not captured in the current model that may account for the variance of the target social-cognitive-belief constructs.

\subsection{Relationship between Perceived Motivation of Health Messages and Social-Cognitive Beliefs}

Regarding the first research question, there is at least a significant relationship between the illness- and/or death-related messages and one of the social-cognitive-belief constructs (see Figure 3). For example, the relationships between the illness-related message ( $\beta=0.11, p<0.01)$ and death-related message $(\beta=0.11, p<0.01)$, on one hand, and outcome expectation, on the other hand, are significant. However, this is not the case with regard to the financial cost-, obesity-, and social stigma-related messages. The implication of this finding is that the more users are motivated by illness-related messages, e.g., "Those who do not find time for exercise will have to find time for illness," the higher their outcome expectations about exercise become. Examples of their outcome expectations include believing that exercise will improve their ability to perform daily activities and overall body functioning. Similarly, the relationships between the illness-related message ( $\beta=0.15$, $p<0.01)$ and death-related message $(\beta=0.24, p<0.001)$, on one hand, and self-regulation belief, on the other hand, are significant. However, with regard to financial cost-, obesity-, and social stigma-related messages, there is no significant relationship. An example implication of this finding is that the more users are motivated by death-related messages, e.g., " $6 \%$ of the world's death is caused by physical inactivity," the more likely they are to 
regulate their exercise by acting. Examples of the self-regulated activities users are likely to do when motivated by death-related messages include setting goals and making plans to achieve them. Finally, there is a significant relationship between the illness-related message and self-efficacy belief $(\beta=0.12, p<0.05)$. The implication of this significant relationship is that the more users are motivated by illness-related messages, the more likely they are to have high self-efficacy beliefs. Examples of their self-efficacy beliefs include believing that they can perform exercise even though they are tired, busy, worried, or depressed.

\subsection{Perceived Motivation of Health Messages}

Regarding the second research question, the results show that, regardless of gender, users were more likely to be motivated by illness- and death-related messages than by financial cost-, obesity-, and social stigma-related messages. As shown in Figure 4, both males and females were motivated by illness- and death-related messages $(M>4$ : the neutral value in the Likert scale). However, both genders were not motivated by financial cost-, obesity-, or social stigma-related messages $(\mathrm{M}<4)$. The implication of these findings, coupled with the significant relationship between illness- and death-related messages and the three social-cognitive beliefs, is that persuasive messages related to illness and death are likely to be effective in public health communication. However, as Figure 4 shows, financial cost-, obesity-, and social stigma-related messages are not likely to be effective.

Direct and Personal Messages. Figure 4 shows that participants, regardless of gender, are not motivated by the message related to the financial cost of physical inactivity. One plausible explanation for this is that the financial cost of physical inactivity (portrayed in the money-related health message) is not direct and/or personal. Regarding the message, "Physical inactivity costs Canadian taxpayers $\$ 6.8$ billion a year" [48], the individual participants do not have to pay directly from their pocket. Rather, the cost is indirect, social, or collective, given that it is "taxpayers' money". Nevertheless, further research needs to be done to investigate the effect of direct-cost-related messages compared with indirect-cost-related messages, and personal-cost-related messages compared with publiccost-related messages. Secondly, a plausible explanation for why participants are not motivated by the obesity-related message, "One in four Canadian adults has clinical obesity", is that it is not personal, making them unable to connect directly with it. Another plausible explanation is that the participants were either fit or have become desensitized to obesity-related information, as the obesity problem has become prevalent, with some experts describing it as a global crisis [60]. However, further research ought to be done to investigate these plausible explanations. Finally, a plausible explanation why participants were not motivated by the social-stigma-related message is that the participants might not have actually believed that "The stigma against people with obesity is comparable to that of racial discrimination". Moreover, the message might not have been applicable to most of the participants as they might be physically fit, and/or not obese, and hence were not motivated by the message.

Authority as a Persuasive Strategy. Research shows that people are more likely to respond to persuasive messages if they are coming from authority figures. Authority is one of Cialdini's [61] six principles of persuasion. Prior research [62] shows that people are motivated by the authority principle. Although the illness- and death-related messages are not direct or personal, they resonate with the participants, as shown in Figures 3 and 4. One plausible explanation is that illness is something that the participants experience in their daily life, personally and/or vicariously. The same explanation also applies to death. Although the message, " $6 \%$ of the world's death is caused by physical inactivity", is not direct or personal, it tends to resonate with the participants as illness does. A plausible explanation is that death is something that everyone can relate to [63]. Nearly everyone would have known a friend or family member who has passed away due to non-communicable diseases caused by physical inactivity, e.g., hypertension, diabetes, or stroke. Another plausible explanation for the effectiveness of the illness- and death-related messages is the reputation of the source. The illness-related message, "Those who do not 
find time for exercise will have to find time for illness", is attributed to Edward Stanley, Earl of Derby (1826-93), who was a British statesman [51]. Similarly, the death-related message, " $6 \%$ of the world's death is caused by physical inactivity," is attributed to World Health Organization, which is perceived as a global authority on public health issues [50]. Edward Stanley and the World Health Organization can be regarded as a figure and body of authority, respectively, which could have had an impact on the participants' perceived motivation of the respective messages. Although one can argue that a good number of the participants might not have known who Edward Stanley was (given that he was not identified as a British statesman in the survey), this may not be the case for the World Health Organization, which is familiar to so many people around the world. Nevertheless, further work needs to be done to investigate the impact of authority figures and bodies on the effectiveness of health messages, and the relationship the messages have with users' social-cognitive beliefs about healthy behaviors.

5.3. Gender Differences in the Relationship between Perceived Motivation of Health Messages and Users' Social-Cognitive Beliefs about Bodyweight Exercise

To answer the third research question, I carried out a multigroup analysis (Table 3). The results of the multigroup analysis showed that gender moderates the relationship between the social stigma-related message and self-efficacy belief/outcome expectation $(p<0.05)$. In the gender-based models for self-efficacy belief, the social stigma-related message was significant in the female model $(\beta=0.17, p<0.05)$, but non-significant in the male model $(\beta=0.02, p>0.05)$. Similarly, in the gender-based models for outcome expectation, the social stigma-related message was significant in the female model $(\beta=0.14$, $p<0.01)$, but non-significant in the male model $(\beta=0.08, p>0.05)$. These findings suggest that messages highlighting the social stigma placed on obesity in society are only likely to be effective in influencing the social-cognitive beliefs about exercise among females. One plausible explanation why the social-stigma-related message has a significant relationship with the social-cognitive beliefs of females is that they are more likely than males to be discriminated against due to obesity, especially in the workplace [64-66]. In a Yale study [67], conducted in the United States, it was found that "women are twice as likely as men to report weight discrimination". The study also found that weight bias is as common and prevalent as racial discrimination [67].

\subsection{Differences in Levels of Perceived Motivation of Health Messages of Different Types}

Regarding the fourth research question, Figure 4 shows that there is no gender difference between males and females in terms of the perceived motivation of each of the five health messages. However, users' perceived motivation of health messages varies across type. Specifically, users are more likely to be motivated by illness- and death-related messages $(M>4)$ than by financial cost-, obesity-, and social stigma-related messages $(\mathrm{M}<4)$.

\subsection{Summary and Implications of Main Findings in the Design of Persuasive Health Messages}

The main findings of the study of the perceived motivation of health messages, and the relationship between them and social-cognitive beliefs about exercise are summarized as follows:

1. Users are motivated by illness- and death-related health messages, but not by financial cost-, obesity-, and social stigma-related messages.

2. There is a significant relationship between the perceived motivation of illness- and death-related messages and users' social-cognitive beliefs about exercise.

3. The more users are motivated by illness- and/or death-related messages, the more likely they are to have high outcome expectations, believe in their ability to perform a health behavior, and regulate themselves towards achieving the health goals.

4. Due to the above findings, Illness- and death-related messages may be used as a persuasive technique to motivate behavior change in persuasive health communication. 
5. Social stigma-based messages related to obesity are likely to be effective in influencing the social-cognitive beliefs of females, but not those of males.

It is noteworthy that, while some prior studies, especially on smoking cessation and risky sexual behavior, have found that self-threatening messages related to mortality can be a barrier to acknowledging health risk [43-45], in the current study in the physical activity domain, this was not the case. The relatively high perceived motivation levels of the illness- and death-related messages and their significant relationship with at least two of the social-cognitive beliefs, are an indication that negative health messages, which reflect loss of health and/or life, may be effective in bringing about behavior change in the exercise domain. This finding is in line with prior findings of studies focused on death-related health messages. For example, in the smoking cessation domain, Martin and Kamins [43] found that that "highlighting family members' inability to cope when one dies can result in health messages that facilitate the acknowledgment of the health risk and reduce risky health behaviors" (p. 1). However, in the physical activity domain, due to the limitations of the current study, further, more systematic studies ought to be carried out to confirm and/or validate the current findings.

Moreover, although there is a significant relationship between the social stigmarelated message and outcome expectation/self-efficacy beliefs among females. However, Figure 4 shows that females, just as males, may not be motivated by stigma-related messages $(M<4)$. Hence, messages related to social stigma may be discouraged in persuasive health communication as they are not likely to be effective in motivating users. The same applies to financial cost- and obesity-related messages, as I found no empirical evidence on their relationship with users' social-cognitive beliefs about exercise. Rather, intervention designers should focus more on messages related to illness and its correlates, e.g., non-communicable diseases caused by physical inactivity, and statistics and figures about physical inactivity and mortality. These types of messages are more likely to be effective as Figures 3 and 4 show. Moreover, apart from sending these types of messages to users, they should also be supported in other ways, e.g., suggested or recommended exercises to perform, provided with tips, and motivated by the beneficial outcomes of physical activity [68].

\subsection{Contributions}

The study makes a number of contributions to the body of knowledge. It adds new findings to the growing literature of fear appeals [43]. Unlike prior research findings that discourage the use of threat-based health messages in the smoking, texting-while-driving, and risky sexual behavior domains [43-45], the current study demonstrates otherwise. It shows that threat-based health messages related to illness and death hold potential in the fitness app domain in terms of increasing the perceived self-efficacy, self-regulation, and outcome expectations of observers of exercise models. This study is the first to model the relationship between users' perceived motivation of health messages and social-cognitive beliefs in the context of behavior modeling. Second, it is the first to show the gender difference in the relationship between users' perceived motivation of health messages and social-cognitive beliefs, which, research has shown, are significant determinants of behavior change. Finally, it is the first to show that there is no significant difference between males' and females' social-cognitive belief profiles with regard to the perceived motivation of the different types of health messages investigated in the study. Particularly, it shows that, regardless of gender, people are more likely to be motivate by illness-related messages, followed by death-related messages, but are not likely to be motivated by financial cost-, obesity-, and social stigma-related messages.

\subsection{Limitations and Future Work}

The study is not devoid of limitations. The first limitation is that the study is based on perception and not an actual fitness app; hence, the findings may not generalize to real-life settings where participants used the app prior to completing the questionnaire. The second 
limitation is that the investigated population samples are residents and/or citizens of Canada and the United States, which are mainly composed of Caucasians (whites). This may threaten the generalizability of the findings to other demographics, countries, and cultures. Hence, there is a need for further studies to investigate the generalizability of the current findings to different user groups. The third limitation of the study is the ecological validity of the questionnaire. Hence, the current findings should be taken with some caution, as they may not generalize to real-life settings or certain populations. For example, although all the SCT-related questions have been validated in prior studies, some may not be relevant to some of the participants in a real-life setting, for example, "I will make my goal public by telling others about it". Regarding this question, some of the participants may not be exercising and setting exercise goals at the time of the study; hence, "making my goal public" would not be relevant to this group of participants. Hence, there is a need for further studies to uncover how this group of participants differs from active participants (who set exercise goals) with regard to the perceived motivation of health messages and its relationship with social-cognitive beliefs. The fourth limitation of the study is that it only employ a single item to measure the health message constructs. Although research has shown that single-item constructs can be as reliable as multi-item constructs [69,70], there may be the need for further studies that explore the use of multiple items to measure the message constructs in question to find out the generalizability of the current findings.

Moreover, the fifth limitation of the study is that it did not investigate how, apart from gender, other demographic variables such as age, culture, race, and education influence the findings. For example, the relationships between perceived motivation of health messages and social-cognitive beliefs in the path models may be different for different age groups (young and old people), different races (black and white), and different levels of education (low and high). Hence, further research needs to be carried out to investigate the moderating effect of these demographic variables. The sixth limitation of the study is that its findings may have been confounded by other variables (related to the messages) not investigated in the current study, e.g., source of the message (authorities vs. non-authorities), the wording of the message (e.g., provision of statistics and figures vs. qualitative information), framing of the message (positive vs. negative), informational appeal (rational vs. emotional), effect of messages (short-term vs. long-term). Particularly, the study did not take into consideration the likelihood of people getting used to messages (e.g., illness- and death-related messages) as they hear or see them again and again, which may make their effect fade away over time. The seventh limitation of the study is that it did not consider other types or variations of messages that may be effective in promoting exercise or may have relationships with social-cognitive beliefs. For example, the study did not include social loss or suffering of loved ones due to illness/death arising from sedentary lifestyle or lack of physical activity [43]. Future research in this area can take on addressing some of these limitations in a more systematic fashion.

\section{Conclusions}

In this paper, I explored the relationship between users' perceived motivation of health messages and social-cognitive beliefs about exercise, and the moderating effect of gender. The data analysis showed that users, regardless of gender, are motivated by illness- and/or death-related messages, but not by financial cost-, obesity-, and social stigma-related messages. Specifically, the path modeling showed that there is a significant relationship between users' perceived motivation of illness- and/or death-related messages and the three social-cognitive beliefs of interest. The findings revealed that the higher the perceived motivation levels of illness- and/or death-related messages are, the more likely users are to have high outcome expectations about exercise, believe in their ability to perform exercise, and regulate themselves. These findings will enable the targeting of the right health messages to the right audience to motivate behavior change in the physical activity domain. The findings provide a basis for fitness app designers to leverage more of illness- and death-related health messages as a persuasive technique to motivate behavior 
change. In future work, I look forward to investigating the effect of other demographic variables (e.g., age, race, and education) and extending the study to other populations outside Canada and United States. This will help us to uncover how the current findings generalize across different demographics and national populations across the world.

Funding: This research was funded by a Natural Sciences and Engineering Research Council of Canada Discovery Grant (RGPIN-2016-05762).

Data Availability Statement: Not applicable.

Acknowledgments: I would like to thank my supervisor, Julita Vassileva, for the advice and feedback she provided me in the course of my doctoral research, from which this paper derives. This paper was first presented as a poster in the 2019 International Symposium on Human Factors and Ergonomics in Health Care.

Conflicts of Interest: The author declares no conflict of interest. The funders had no role in the design of the study; in the collection, analyses, or interpretation of data; in the writing of the manuscript, or in the decision to publish the results.

\section{References}

1. Public Health Communications. A Public Health Intervention. Available online: https://professionals.wrha.mb.ca/old/extranet/ publichealth/priorities-communication.php (accessed on 31 July 2021).

2. Bandura, A. Health promotion from the perspective of social cognitive theory. Psychol. Health 1998, 13, 623-649. [CrossRef]

3. McCormack, L.; Sheridan, S.; Lewis, M.; Boudewyns, V.; Melvin, K.L.; Kistler, C.; Lux, L.J.; Cullen, K.; Lohr, K.N. Communication and Dissemination Strategies to Facilitate the Use of Health-Related Evidence. Evid. Rep. Technol. Assess. 2013, 1-520. [CrossRef]

4. The Community Guide: Health Communication and Health Information Technology. Available online: https://www. thecommunityguide.org/topic/health-communication-and-health-information-technology (accessed on 1 July 2021).

5. Public Health Agency of Canada. Core Competencies for Public Health in Canada. 2008; pp. 1-25. Available online: https: //www.phac-aspc.gc.ca/php-psp/ccph-cesp/pdfs/cc-manual-eng090407.pdf (accessed on 1 July 2021).

6. Wakefield, M.A.; Loken, B.; Hornik, R.C. Use of mass media campaigns to change health behaviour. Lancet 2010, 376, 1261-1271. [CrossRef]

7. Rimal, R.N.; Lapinski, M.K. Why health communication is important in public health. Bull. World Health Organ. 2009, 87, 247. [CrossRef] [PubMed]

8. Public Health Ontario. Health Communication Message Review Criteria, 2nd ed.; Queen's Printer for Ontario: Toronto, ON, Canada, 2012. Available online: https:/ / www.publichealthontario.ca/-/media/documents/H/2012/health-comm-review-crtieria.pdf (accessed on 20 August 2021).

9. Gallagher, K.M.; Updegraff, J.A. Health Message Framing Effects on Attitudes, Intentions, and Behavior: A Meta-analytic Review. Ann. Behav. Med. 2012, 43, 101-116. [CrossRef] [PubMed]

10. Ryu, S. Book Review: mHealth: New Horizons for Health through Mobile Technologies: Based on the Findings of the Second Global Survey on eHealth (Global Observatory for eHealth Series, Volume 3). Health Inform. Res. 2012, 18, 231-233. [CrossRef]

11. Hall, A.K.; Cole-Lewis, H.; Bernhardt, J.M. Mobile Text Messaging for Health: A Systematic Review of Reviews. Annu. Rev. Public Health 2015, 36, 393-415. [CrossRef]

12. Gowin, M.; Cheney, M.K.; Gwin, S.H.; Wann, T.F. Health and Fitness App Use in College Students: A Qualitative Study. Am. J. Health Educ. 2015, 46, 223-230. [CrossRef]

13. Murnane, E.L.; Huffaker, D.; Kossinets, G. Mobile health apps. In Proceedings of the 2015 ACM International Joint Conference on Pervasive and Ubiquitous Computing and Proceedings of the 2015 ACM International Symposium on Wearable Computers (UbiComp '15), Osaka, Japan, 9-11 September 2015; ACM: New York, NY, USA, 2015; pp. 261-264.

14. Park, K.; Weber, I.; Cha, M.; Lee, C. Persistent Sharing of Fitness App Status on Twitter. In Proceedings of the 19th ACM Conference on Computer-Supported Cooperative Work \& Social Computing, San Francisco, CA, USA, 27 February-2 March 2016; Volume 27, pp. 184-194. [CrossRef]

15. Oyibo, K.; Olagunju, A.H.; Olabenjo, B.; Adaji, I.; Deters, R.; Vassileva, J. BEN'FIT: Design, implementation and evaluation of a culture-tailored fitness app. In Proceedings of the Adjunct Publication of the 27th Conference on User Modeling, Adaptation and Personalization, Larnaca, Cyprus, 6 June 2019; pp. 161-166. [CrossRef]

16. Barkley, J.E.; Lepp, A.; Santo, A.; Glickman, E.; Dowdell, B. The relationship between fitness app use and physical activity behavior is mediated by exercise identity. Comput. Hum. Behav. 2020, 108, 106313. [CrossRef]

17. Keung, C.; Lee, A.; Lu, S.; O'Keefe, M. BunnyBolt: A mobile fitness app for youth. In Proceedings of the 12th International Conference on Interaction Design and Children, New York, NY, USA, 24-27 June 2013; pp. 585-588. [CrossRef]

18. Anderson, E.S.; Wojcik, J.R.; Winett, R.A.; Williams, D.M. Social-cognitive determinants of physical activity: The influence of social support, self-efficacy, outcome expectations, and self-regulation among participants in a church-based health promotion study. Health Psychol. 2006, 25, 510-520. [CrossRef] 
19. Oyibo, K.; Orji, R.; Vassileva, J. Developing Culturally Relevant Design Guidelines for Encouraging Physical Activity: A Social Cognitive Theory Perspective. J. Health Inform. Res. 2018, 2, 319-352. [CrossRef]

20. Oyibo, K.; Adaji, I.; Orji, R.; Olabenjo, B.; Azizi, M.; Vassileva, J. Perceived Persuasive Effect of Behavior Model Design in Fitness Apps. In Proceedings of the 26th Conference on User Modeling, Adaptation and Personalization, Singapore, 8-11 July 2018; pp. 219-228.

21. Watson, A.; Bickmore, T.; Cange, A.; Kulshreshtha, A.; Kvedar, J. An Internet-Based Virtual Coach to Promote Physical Activity Adherence in Overweight Adults: Randomized Controlled Trial. J. Med. Internet Res. 2012, 14, e1. [CrossRef]

22. Vollmer, J.; Schuster, P.; Giuliani, M. Plank Challenge with NAO: Using a Robot to Persuade Humans to Exercise Longer. In Proceedings of the 11th International Conference on Persuasive Technology, Salzburg, Austria, 4-7 April 2016; pp. 50-53.

23. Conroy, D.E.; Yang, C.-H.; Maher, J.P. Behavior Change Techniques in Top-Ranked Mobile Apps for Physical Activity. Am. J. Prev. Med. 2014, 46, 649-652. [CrossRef]

24. Bandura, A. Social Cognitive Theory of Mass Communication. In Media Effects: Advances in Theory and Research; Lawrence Erlbaum: Mahwah, NJ, USA, 2001; pp. 121-153.

25. Bandura, A. Social Foundations of Thought and Action: A Social Cognitive Theory; Prentice-Hall: Englewood Cliffs, NJ, USA, 1986.

26. Fountoukidou, S.; Ham, J.; Matzat, U.; Midden, C. Effects of an artificial agent as a behavioral model on motivational and learning outcomes. Comput. Hum. Behav. 2019, 97, 84-93. [CrossRef]

27. Stibe, A. Socially Influencing Systems: Persuading People to Engage with Publicly Displayed Twitter-Based Systems. 2014. Available online: http:/ / urn.fi/urn:isbn:9789526205410 (accessed on 10 July 2021).

28. Lin, C.-Y.; Chen, Y.-S.; Tsai, J.-C. Exploring the Triple Reciprocity of Information System Psychological Attachment; Springer Science and Business Media LLC: Berlin/Heidelberg, Germany, 2014; pp. 779-785.

29. Fogg, B.J. Persuasive Technology: Using Computers to Change What We Think and Do; Morgan Kaufmann: San Francisco, CA, USA, 2003; pp. 31-65.

30. Oyibo, K.; Adaji, I.; Vassileva, J. Social cognitive determinants of exercise behavior in the context of behavior modeling: A mixed method approach. Digit. Health 2018, 4. [CrossRef]

31. Rovniak, L.S.; Anderson, E.S.; Winett, R.A.; Stephens, R.S. Social cognitive determinants of physical activity in young adults: A prospective structural equation analysis. Ann. Behav. Med. 2002, 24, 149-156. [CrossRef]

32. Bandura, A. Self-Efficacy: The Exercise of Control; Freeman: New York, NY, USA, 1997.

33. Schwarzer, R.; Renner, B. Social-cognitive predictors of health behavior: Action self-efficacy and coping self-efficacy. Health Psychol. 2000, 19, 487-495. [CrossRef]

34. Bandura, A. Social cognitive theory of self-regulation. Organ. Behav. Hum. Decis. Process 1991, 50, 248-287. [CrossRef]

35. Wójcicki, T.; White, S.M.; McAuley, E. Assessing Outcome Expectations in Older Adults: The Multidimensional Outcome Expectations for Exercise Scale. J. Gerontol. Ser. B 2009, 64, 33-40. [CrossRef]

36. Breslin, S.; Wadhwa, B. Gender and Human-Computer Interaction. Wiley Handb. Hum. Comput. Interact. 2017, 1, 71-87. [CrossRef]

37. Boiano, S.; Borda, A.; Bowen, J.; Faulkner, X.; Gaia, G.; McDaid, S. Gender Issues in HCI Design for Web Access. In Advances in Universal Web Design and Evaluation: Research, Trends and Opportunities; Kur-niawan, S., Zaphiris, P., Eds.; Idea Group Publishing: Hershey, PA, USA, 2006; pp. 116-153.

38. Orji, R.; Mandryk, R.; Vassileva, J. Gender and Persuasive Technology: Examining the Persuasiveness of Persuasive Strategies by Gender Groups. In Proceedings of the 9th International Conference on Persuasive Technology, Padova, Italy, 21 May 2014; pp. $48-52$.

39. Oyibo, K.; Orji, R.; Vassileva, J. The Influence of Culture in the Effect of Age and Gender on Social Influence in Persuasive Technology. In Proceedings of the Adjunct Publication of the 25th Conference on User Modeling, Adaptation and Personalization, Bratislava, Slovakia, 12 July 2017; ACM: New York, NY, USA,; pp. 47-52.

40. Abdullahi, A.M.; Oyibo, K.; Orji, R.; Kawu, A.A. The Influence of Age, Gender, and Cognitive Ability on the Susceptibility to Persuasive Strategies. Information 2019, 10, 352. [CrossRef]

41. Oyibo, K.; Vassileva, J. Investigation of persuasive system design predictors of competitive behavior in fitness application: A mixed-method approach. Digit. Health 2019, 5. [CrossRef] [PubMed]

42. Dağgöl, G.D. Perceived Academic Motivation and Learner Empowerment Levels of EFL Students in Turkish Context. Particip. Educ. Res. 2020, 7, 21-37. [CrossRef]

43. Martin, I.M.; Kamins, M.A. Effectively using death in health messages: Social loss versus physical mortality salience. J. Consum. Behav. 2019, 18, 205-218. [CrossRef]

44. Sherman, D.K.; Cohen, G.L. Accepting Threatening Information: Self-Affirmation and the Reduction of Defensive Biases. Curr. Dir. Psychol. Sci. 2002, 11, 119-123. [CrossRef]

45. Goldenberg, J.L.; Arndt, J. The implications of death for health: A terror management health model for behavioral health promotion. Psychol. Rev. 2008, 115, 1032-1053. [CrossRef]

46. Oyibo, K.; Vassileva, J. Investigation of the moderating effect of race-based personalization of behavior model design in fitness application. SN Appl. Sci. 2019, 1, 1118. [CrossRef]

47. Block, L.G.; Keller, P.A. When to Accentuate the Negative: The Effects of Perceived Efficacy and Message Framing on Intentions to Perform a Health-Related Behavior. J. Mark. Res. 1995, 32, 192. [CrossRef] 
48. CBCNews. Physical Inactivity Costs Taxpayers 6.8B a Year; Canadian Press: Toronto, ON, Canada, 2012. Available online: https://www.cbc.ca/news/health/physical-inactivity-costs-taxpayers-6-8b-a-year-1.1134811 (accessed on 20 August 2021).

49. Hodgson, C.; Corscadden, L.; Taylor, A.; Sebold, A.; Pearson, C.; Kwan, A.; Sommerer, S.; Halley, R.L.; Walsh, P.; Shane, A.; et al. Obesity in Canada: A joint report from the Public Health Agency of Canada and the Canadian Institute for Health Information. Can. Inst. Health Inf. 2011, 62, 1-54.

50. World Health Organization. Global Recommendations on Physical Activity for Health; World Health Organization: Geneva, Switzerland, 2010.

51. Lavie, C.J.; Thomas, R.J.; Squires, R.W.; Allison, T.G.; Milani, R.V. Exercise training and cardiac rehabilitation in primary and secondary prevention of coronary heart disease. Mayo Clin. Proc. 2009, 84, 373-383. [CrossRef]

52. Obesity in Canada. Available online: https://obesitycanada.ca/obesity-in-canada/ (accessed on 20 August 2021).

53. McGaan, L. Introduction to Persuasion, Monmouth College. Available online: https://department.monm.edu/cata/rankin/ Classes/Scat101/assignments/rational.htm (accessed on 20 August 2021).

54. Buhrmester, M.D.; Kwang, T.; Gosling, S. Amazon's Mechanical Turk. Perspect. Psychol. Sci. 2011, 6, 3-5. [CrossRef]

55. Sanchez, G. PLS Path Modeling with R. 2013. Available online: https://www.gastonsanchez.com/PLS_Path_Modeling_with_R. pdf (accessed on 20 August 2021).

56. Hair, J.F.; Hult, G.T.M.; Ringle, C.M.; Sarstedt, M. A Primer on Partial Least Squares Structural Equation Modeling (PLS-SEM); Sage Publications, Inc.: Washington, DC, USA, 2014.

57. Hussain, S.; Fangwei, Z.; Siddiqi, A.F.; Ali, Z.; Shabbir, M.S. Structural Equation Model for Evaluating Factors Affecting Quality of Social Infrastructure Projects. Sustainability 2018, 10, 1415. [CrossRef]

58. Dunn, T.J.; Baguley, T.; Brunsden, V. From alpha to omega: A practical solution to the pervasive problem of internal consistency estimation. Br. J. Psychol. 2013, 105, 399-412. [CrossRef]

59. Peters, G. userfriendlyscience: Quantitative Analysis Made Accessible. R Package. Version 0.3-0. 2015. Available online: https://rdrr.io/cran/userfriendlyscience/ (accessed on 20 August 2021).

60. Birrell, I. Obesity: Africa's New Crisis. Available online: http://www.theguardian.com/society/2014/sep/21/obesity-africasnew-crisis (accessed on 10 July 2020).

61. Cialdini, R.B. Influence: The Psychology of Persuasion, revised ed.; Harper Collins: New York, NY, USA, 2006.

62. Oyibo, K.; Adaji, I.; Orji, R.; Olabenjo, B.; Vassileva, J. Susceptibility to persuasive strategies: A comparative analysis of Nigerians vs. Canadians. In Proceedings of the 26th Conference on User Modeling, Adaptation and Personalization-UMAP 2018, Singapore, 8-11 July 2018; pp. 229-238.

63. Keeley, M.P. Family Communication at the End of Life. Behav. Sci. 2017, 7, 45. [CrossRef]

64. Puhl, R. Weight Discrimination: A Socially Acceptable Injustice. Available online: http://www.obesityaction.org/wp-content/ uploads/Obesity-Discrimination.pdf (accessed on 15 May 2020).

65. Andreyeva, T.; Puhl, R.M.; Brownell, K.D. Changes in Perceived Weight Discrimination among Americans, $1995-1996$ through 2004-2006. Obesity 2008, 16, 1129-1134. [CrossRef]

66. Puhl, R.; Brownell, K. Bias, Discrimination and Obesity. Handb. Obes. 2008, 461-470, 81-89. [CrossRef]

67. Rudd Center for Food Policy \& Obesity. Yale Study Shows Weight Bias Is as Prevalent as Racial Discrimination. Available online: https:/ /news.yale.edu/2008/03/27/yale-study-shows-weight-bias-prevalent-racial-discrimination (accessed on 12 September 2020).

68. Oyibo, K. Designing Culture-Tailored Persuasive Technology to Promote Physical Activity. 2020. Available online: https: / harvest.usask.ca/handle/10388/12943 (accessed on 21 January 2021).

69. Spörrle, M.; Bekk, M. Meta-Analytic Guidelines for Evaluating Single-Item Reliabilities of Personality Instruments. Assessment 2013, 21, 272-285. [CrossRef]

70. Bergkvist, L.; Rossiter, J.R. The Predictive Validity of Multiple-Item versus Single-Item Measures of the Same Constructs. J. Mark. Res. 2007, 44, 175-184. [CrossRef] 